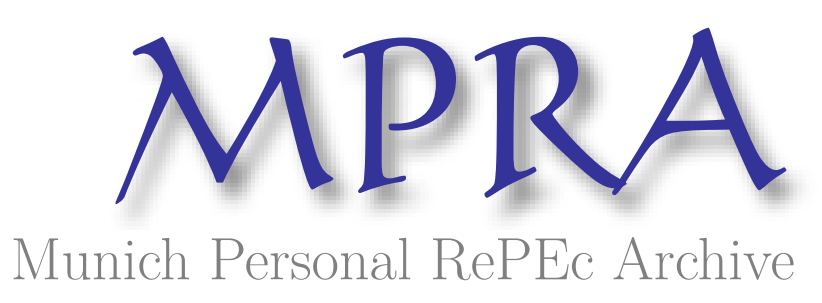

\title{
Forecasting ENSO with a smooth transition autoregressive model
}

Ubilava, David and Helmers, C Gustav

January 2012

Online at https://mpra.ub.uni-muenchen.de/36890/

MPRA Paper No. 36890, posted 24 Feb 2012 14:29 UTC 


\title{
Forecasting ENSO with a Smooth Transition Autoregressive \\ Model
}

\author{
David Ubilava $^{\mathrm{a} *} \quad$ C. Gustav Helmers ${ }^{\mathrm{b}}$ \\ ${ }^{\text {a }}$ Post Doctoral Researcher. Department of Agricultural Economics, Mississippi State University. PO Box \\ 5187 Mississippi State, MS 39762, USA. Tel: +1 662325 2676; Fax: +1 662325 8777; E-mail: \\ ubilava@agecon.msstate.edu \\ b Assistant Professor. Hutson School of Agriculture, Murray State University. 213 South Oakley Applied \\ Science Murray, KY 42071, USA. Tel: +1 270809 6932; Fax: +1 270809 3441; E-mail: \\ chelmers@murraystate.edu
}

\begin{abstract}
This study examines the benefits of nonlinear time series modelling to improve forecast accuracy of the El Niño Southern Oscillation (ENSO) phenomenon. The paper adopts a smooth transition autoregressive (STAR) modelling framework to assess the potentially regime-dependent dynamics of sea surface temperature anomaly. The results reveal STAR-type nonlinearities in ENSO dynamics, resulting in superior out-of-sample forecast performance of STAR over the linear autoregressive models. The advantage of nonlinear models is especially apparent in the short- and intermediate-term forecasts. These results are of interest to researchers and policy makers in the fields of climate dynamics, agricultural production, and environmental management.
\end{abstract}

Keywords: El Niño Southern Oscillation; Out-of-Sample Forecasting; Smooth Transition Autoregression

\footnotetext{
${ }^{*}$ Corresponding author
} 


\section{Introduction}

In recent years there has been growing interest in studying the role of weather anomalies on the performance of various economic variables (Keppenne, 1995; Brunner, 2002; Kim and McCarl, 2005). Researchers have paid particular attention to the large-scale medium-frequency event known as El Niño Southern Oscillation (ENSO) (e.g. Glantz, 2001), and its economic effects (e.g. Handler, 1990; Carlson et al., 1996; Hansen et al., 1998; Legler et al., 1999). Studies have shown that the ENSO impact on the world economy is significant. For example, some of the extreme episodes of ENSO have caused damages exceeding several billions of dollars in the United States alone (Adams et al., 1999; Pielke Jr and Landsea, 1999). Additionally, studies have shown that ENSO impact goes beyond its economic importance, and affects social lives too, to the extent that it may even cause civil conflicts in the developing world (Hsiang et al., 2011). The corollary is also true: society will benefit from improved prediction of extreme ENSO events, as it will give economic agents and policy makers time to plan ahead. For example, the expected annual value of accurate ENSO prediction in the agricultural sector of the U.S. has been measured to be in the order of several hundred million dollars (Solow et al., 1998).

What the foregoing discussion shows is that ENSO is an important and costly phenomenon, and more accurate forecasts of this anomaly has the potential of mitigating social and economic costs associated with it. A variety of methods have been proposed and used to predict ENSO occurrences, many of which rely on historical behavior of the time series data (Kirtman and Schopf, 1998; Tang and Hsieh, 2002; Halide and Ridd, 2008; Deng and Tang, 2009). In this context, studies have shown that ENSO cycles can be associated with nonlinear dynamics (e.g. An and Jin, 2004; Boucharel et al., 2011), thus one obvious way to improve the ENSO forecasting accuracy is by implementing 
more sophisticated, nonlinear time series models. Recent developments in nonlinear time series modelling have allowed researchers to examine the intricate dynamics of an ENSO cycle (e.g. Tangang et al., 1998; Berliner et al., 2000; Hall et al., 2001; An, 2009). In this research we adopt the smooth transition autoregressive (STAR) modelling framework of (Luukkonen et al., 1988) and (Terasvirta, 1994) to investigate regime-dependent asymmetries in ENSO dynamics. STAR models have been advocated for their superiority in fitting turbulent variable dynamics. This modelling framework thus appears to be a suitable method to analyze ENSO dynamics, and has been successfully applied by (Hall et al., 2001), who demonstrate support for distinct autocorrelations in ENSO anomalies in different, El Niño and La Niña regimes. Based on these findings, they suggest that point forecasts from nonlinear models are likely to outperform those from linear models. The focal point of the current research is finding whether the improvement in in-sample fit corresponds to more accurate out-of-sample prediction.

The objective of this study is to assess ENSO forecast accuracy from STAR-type nonlinear models. We hypothesize that parameters of nonlinear models facilitate improved forecast accuracy over their linear counterparts. Using historical monthly SST data we generate a sequence out-of-sample forecasts from linear and nonlinear models, and examine them with respect to actual realizations of SST. By means of statistical tests and graphical illustrations we derive inferences about forecast performance of nonlinear and linear models. In what follows, we first present the methodological framework, describing the peculiarities of STAR modelling and forecasting. We then proceed by implementing this technique in the empirical framework, where we provide information regarding the data used in this research, and discuss main results of this study, followed by summarizing our findings in the conclusions. 


\section{Modelling Framework}

The general idea behind time series modelling is that a current observation is a function of its previously observed values. A basic representation of this relationship is a linear autoregressive (AR) model, which may be expressed in first differenced form as in Equation (1).

$$
\Delta y_{t}=\theta^{\prime} x_{t}+\varepsilon_{t}
$$

where $\Delta$ is a first-difference operator, such that $\Delta y_{t}=y_{t}-y_{t-1} ; y_{t}$ is a dependent

variable; $x_{t}=\left(1, y_{t-1}, \Delta y_{t-1}, \ldots, \Delta y_{t-p+1}\right)^{\prime}$ is a vector of right-hand-side variables; $\theta=\left(\alpha, \beta, \phi_{1}, \ldots, \phi_{p-1}\right)^{\prime}$ is a vector of parameters to be estimated, where $\beta$ is the unit root parameter, such that the restriction $\beta=0$ imposes a unit root process; finally, $\varepsilon_{t}$ is an additive error process such that $\varepsilon_{t} \sim i i d\left(0, \sigma^{2}\right)$.

By relaxing the linearity assumption, Equation (1) can be further augmented in a number of ways, resulting in several well-known nonlinear frameworks such as threshold autoregressive (e.g. Tsay, 1989; Tong, 1990), Markov switching (e.g. Hamilton, 1989), and artificial neural network (e.g. Kuan and White, 1994) models. An alternative specification, which is also a nonlinear generalizations of a basic AR model, and moreover which embeds elements of the aforementioned nonlinear models, is the STAR model. Conceptually, smooth transition regressions were first proposed by Bacon and Watts (1971), while modelling and testing methods were subsequently developed by Luukkonen et al. (1988), Terasvirta and Anderson (1992), Terasvirta (1994), and Eitrheim and Terasvirta (1996).

STAR-type models are widely applied in studies modelling asymmetric cyclical variations(e.g. Terasvirta, 1995; Hall et al., 2001). One of the attractive features of the 
STAR modelling framework is that it it allows for non-discrete switching points between the extreme regimes, resulting in a potentially smooth transition between them. Since its introduction, the STAR modelling approach has gained in popularity and has been increasingly applied to examine potential nonlinearities of unemployment rates, GDP, money demand, and interest rates (e.g. Terasvirta, 1995; Eitrheim and Terasvirta, 1996; Sarantis, 1999; Skalin and Terasvirta, 2002), and more recently to investigate nonlinear features of climate variables, including ENSO (Hall et al., 2001), and their effects on commodity prices (Ubilava, 2012).

The general class of smooth transition regressions can be specified as Equation (2).

$$
\Delta y_{t}=\theta_{0}{ }^{\prime} x_{t}+\theta_{1}{ }^{\prime} x_{t} G\left(s_{t} ; \gamma, c\right)+\varepsilon_{t}
$$

where $G\left(s_{t} ; \gamma, c\right)$ represents the so called transition function, which is, by construction, bounded between zero and one, and where $s_{t}$ is a transition variable, and $\gamma$ and $c$ are, respectively, smoothness and location parameters. The transition function alters the dynamics of the model conditional on the transition variable in a potentially smooth manner.

A generalized version of one of the more frequently applied transition functions is represented as Equation (3):

$$
G\left(s_{t} ; \gamma, \mathbf{c}\right)=\left\{1+\exp \left[-\gamma / \sigma_{s_{t}}^{k} \prod_{k}\left(s_{t}-c_{k}\right)^{k}\right]\right\}^{-1}
$$

where $\sigma_{s_{t}}$ is the standard deviation of the transition variable. By setting $k=1$ and $k=2$, one obtains logistic and quadratic transition functions, respectively, resulting in logistic STAR (LSTAR) and quadratic STAR (QSTAR) models. An alternative transition function is an exponential function, yielding an exponential STAR (ESTAR) model, and 
defined as Equation (4).

$$
G\left(s_{t} ; \gamma, c\right)=\left\{1-\exp \left[-\gamma / \sigma_{s_{t}}^{2}\left(s_{t}-c\right)^{2}\right]\right\}
$$

In the transition functions the smoothness parameter, $\gamma$, is defined to be a non-negative parameter. The LSTAR and QSTAR models converge to a linear AR model when $\gamma \rightarrow 0$, and a threshold autoregressive (TAR) model when $\gamma \rightarrow \infty$. Alternatively, ESTAR converges to a linear AR model both when either $\gamma \rightarrow 0$ or $\gamma \rightarrow \infty$.

\subsection{Testing Nonlinearities}

The question of whether nonlinearity is truly an underlying feature of the data is a testable hypothesis. However, we cannot directly test the null hypothesis of linearity, that is, $H_{0}: \gamma=0$, in a STAR model, due to unidentified nuisance parameters, also known as Davies' problem (Davies, 1977, 1987). Specifically, in the context of Equation (2), the nonlinear model will reduce to the linear AR model by imposing the restriction $\gamma=0$ or $\theta_{1}=0$. Therefore, the standard test statistics are no longer applicable. Luukkonen et al. (1988) proposed a solution to the problem by approximating the transition function,

$G\left(s_{t} ; \gamma, c\right)$, using a third order Taylor series expansion. This results in a testable auxiliary regression, expressed as Equation (5):

$$
\Delta y_{t}=\theta_{0}{ }^{\prime} x_{t}+\sum_{i=1}^{3} \theta_{i}{ }^{\prime} x_{t} s_{t}^{i}+\xi_{t}
$$

where $\xi_{t}$ combines the original error term, $\varepsilon_{t}$, and the approximation error resulting from the Taylor expansion. The new specification makes it possible to apply conventional testing methods, particularly in the test for linearity against the STAR specification. This is now equivalent to testing the null hypothesis of $H_{0}^{\prime}: \theta_{1}=\theta_{2}=\theta_{3}=0$, where $\theta_{i}$, 
$i=1,2,3$, are vectors of parameters from the auxiliary regression. An additional benefit of testing nonlinearities using a third order approximation, as presented in Equation (5), is that tests against LSTAR and ESTAR/QSTAR models are also embedded in the testing framework: the test against the LSTAR model is equivalent to testing the null hypotheses of $H_{03}: \theta_{3}=0$ and $H_{01}: \theta_{1}=0 \mid \theta_{2}=\theta_{3}=0$. Alternatively, the test against the ESTAR/QSTAR is equivalent to $H_{02}: \theta_{2}=0 \mid \theta_{3}=0$.

In practice, the transition variable is often a priori unknown. One is, therefore, required to test a set of candidate transition variables, and select the suitable transition variable based on probability values associated with the aforementioned hypotheses. Once the transition variable (and the associated transition function) is selected, one may proceed to estimate the related STAR model using a nonlinear optimization procedure (refer to Luukkonen et al. (1988); Terasvirta (1995); Eitrheim and Terasvirta (1996) for additional details regarding the testing procedure, including remaining nonlinearity, parameter constancy and residual autocorrelation tests withing the STAR modelling framework).

\subsection{Out-of-Sample Forecasting}

Forecast accuracy improvement is assessed by analyzing the relative out-of-sample forecast performance of the linear (restricted) and the nonlinear (unrestricted) models as represented by Equations (1) and (2), respectively. Consider a general specification of a univariate autoregression given as Equation (6).

$$
y_{t}=f\left(x_{t} \mid \theta\right)+\varepsilon_{t}
$$

where the dependent variable, $y_{t}$, is defined by a linear or nonlinear function of the

right-hand-side variables, $x_{t}=\left(y_{t-1}, \ldots, y_{t-p}, \ldots\right)^{\prime}$, and an error term, $\varepsilon_{t}$. Based on 
Equation (6), the expected one-step-ahead forecast can be written as Equation (7).

$$
y_{t+1 \mid t}^{f}=E\left(y_{t+1 \mid t}\right)=E\left(f\left(x_{t+1} \mid \theta\right)+\varepsilon_{t+1}\right)=E\left(f\left(x_{t+1} \mid \theta\right)\right)
$$

where $x_{t+1}=\left(y_{t}, \ldots, y_{t-p+1}, \ldots\right)^{\prime}$. In the case of linear models, an $h$-step-ahead forecast, where $h>1$, may be obtained recursively in a manner similar to Equation (7). This is a naïve, or so called "skeleton extrapolation" approach, which in the case of nonlinear models would yield biased forecasts (see Tong, 1990; Granger and Terasvirta, 1993). Alternatively, a proper forecasting method would require a numerical integration, which quickly becomes cumbersome as the forecast horizon length increases. This can be circumvented by approximating the multidimensional integral using a simulation or a bootstrap resampling method (e.g. Lundbergh and Terasvirta, 2004). For example, in the case of $h=2$, the bootstrap forecast of $y_{t+2}$ yields Equation (8).

$$
y_{t+2 \mid t}^{f b}=E\left(y_{t+2 \mid t}^{b}\right)=E\left(f\left(x_{t+2, b} \mid \theta\right)+\varepsilon_{t+2}\right)=B^{-1} \sum_{b=1}^{B}\left(f\left(x_{t+2, b} \mid \theta\right)\right)
$$

where for $p>2, x_{t+2}=\left(y_{t+1 \mid t}^{b}+\varepsilon_{t+1}, y_{t}, \ldots, y_{t-p+2}, \ldots\right)^{\prime}$, and where the idiosyncratic shocks, $\varepsilon_{t+j, b}, j=1,2$, are randomly drawn from the distribution of residuals of the estimated nonlinear model. A general $h$-step-ahead forecast equation, Equation (9), can thus be developed in a manner similar to Equation (8).

$$
y_{t+h \mid t}^{f b}=E\left(y_{t+h \mid t}^{b}\right)=E\left(f\left(x_{t+h, b} \mid \theta\right)+\varepsilon_{t+h}\right)=B^{-1} \sum_{b=1}^{B}\left(f\left(x_{t+h, b} \mid \theta\right)\right)
$$

By applying the bootstrap resampling method one can effectively generate a distribution of forecasts around the mean. Therefore, this approach can also be useful if one intends to calculate empirical confidence intervals of the forecasts. 


\subsubsection{Out-of-Sample Forecast Evaluation}

Out-of-sample forecasts are evaluated using some forecast accuracy measures, one of which is Root Mean Squared Forecast Errors (RMSFE) defined as Equation (10).

$$
\mathrm{RMSFE}=\left(R^{-1} \sum_{r=1}^{R}\left|y_{r, t+h}-y_{r, t+h \mid t}^{f b}\right|^{2}\right)^{\frac{1}{2}}
$$

where $y_{r, t+h}$ is the actual realization of the event at the horizon $h$, and $R$ is the total number of out-of-sample forecasts.

The statistical significance of the forecast accuracy may be examined using loss differential approach, originally proposed by Diebold and Mariano (1995) and subsequently modified by Harvey et al. (1997). The loss differential for an observation $t$ is defined by $d_{t}=g\left(e_{i, t, h}\right)-g\left(e_{j, t, h}\right)$, where $g(\cdot)$ in the current case is a quadratic loss function, and $e_{i, t, h}$ and $e_{j, t, h}$ are $h$-step-ahead forecast error functions for two methods $i$ and $j$ to be compared. Thus, the null hypothesis of equal forecast performances of the two competing models, in terms of the forecast loss functions, is $H_{0}^{\mathrm{d}}: \bar{d}=0$, where $\bar{d}=n^{-1} \sum_{t=1}^{n} d_{t}$, and where $n$ is the total number of loss differentials.

The original Diebold and Mariano (1995) version of the loss differential statistic, DM, assumes that $\bar{d}$ is asymptotically normally distributed. However, we implement a small-sample version of the DM statistic (Harvey et al., 1997). The modified Diebold-Mariano (M-DM) statistic is defined as in Equation 11, where $h$-step-ahead forecasts are assumed to be autocorrelated up to and including the lag $h-1$, but not at lag $h$ and beyond:

$$
\mathrm{M}-\mathrm{DM}=\left\{n^{-1}\left[n+1-2 h+n^{-1} h(h-1)\right]\right\}^{1 / 2} \mathrm{DM}
$$


where

$$
\mathrm{DM}=[V(\bar{d})]^{-1 / 2} \bar{d}
$$

and where $V(\bar{d})$ is the variance of the mean loss differential as defined in Equation (13):

$$
V(\bar{d})=n^{-1}\left(\gamma_{0}+2 \sum_{k=1}^{h-1} \gamma_{k}\right)
$$

where

$$
\gamma_{k}=n^{-1} \sum_{t=k+1}^{n}\left(d_{t}-\bar{d}\right)\left(d_{t-k}-\bar{d}\right) ; \quad k=0, \ldots, h-1
$$

Not uncommonly, this specification of the variance, as presented in Equation 13, may result in a negative value. To circumvent this problem, the variance equation is modified (Equation (15)) using a weighting approach proposed by Newey and Kenneth (1987):

$$
V^{*}(\bar{d})=n^{-1}\left(\gamma_{0}+2 \sum_{k=1}^{h-1} w_{k} \gamma_{k}\right)
$$

where $w_{k}=(1-k / h)$ is a weight associated with the $k^{t h}$ autocovariate. Finally, the obtained M-DM statistic follows Student's t-distribution with $(n-1)$ degrees of freedom.

\section{Empirical Framework}

In this section we apply the methodology outlined in the previous section to obtain estimates for the linear and nonlinear models. These estimates are then used to forecast ENSO and perform an out-of-sample forecast accuracy test. 


\subsection{Data}

The sample consists of monthly observations between January 1950 and December 2011. The time series variable representing the ENSO anomaly, Niño 3.4, is derived from the index tabulated by the Climate Prediction Center at the National Oceanic and Atmospheric Administration. This index measures the difference in Sea Surface Temperature (SST) in the area of the Pacific Ocean between $5^{\circ} N-5^{\circ} S$ and $170^{\circ} \mathrm{W}-120^{\circ} \mathrm{W}$, and is therefore a strong indicator of ENSO occurrence in the tropical Pacific. The Niño 3.4 monthly measure is an average of daily values interpolated from weekly measures obtained from both satellites and actual locations around the Pacific. The anomaly is the deviation of the Niño 3.4 monthly measure from the average historic measure for that particular month from the period $1971-2000$.

In order to generate out-of-sample forecasts, we employ an expanding window modelling approach (e.g. Terasvirta et al., 2005). The benefit of this approach, as opposed to a fixed-length rolling window approach (e.g. Milas and Rothman, 2008), is that at any point of time we employ the maximum historical information available. On the other hand, the potential drawback of this approach is the de-emphasized possibility of structural breaks (see Swanson, 1998; Terasvirta et al., 2005). However, we took advantage of parameter constancy tests in STAR testing framework to find out that there is indeed no evidence of a structural change in any of the windows employed. So, we proceeded by estimating STAR models using an expanding window framework. We simulate a set of $h$-step-ahead forecasts for each window, where the maximum horizon length, $h_{\max }$, is set to 36 months. The first estimation window covers the period from January of 1952 to December of 1990, and the last window ends with December of 2008. Within this range, each consecutive window is estimated by expanding the window ahead by one observation, which in our case constitutes one month. This approach generates a 
total of $216 h$-step-ahead forecasts, $h=1, \ldots, h_{\max }$. Finally, while the models are re-estimated on a monthly basis, the autoregressive lag structure and the delay lag of the transition variable are re-examined on an annual basis.

\subsection{Estimation Results}

We use the Augmented Dickey-Fuller (ADF) test to examine the stationarity hypothesis of the ENSO variable. Based on the test results, we reject the unit root in the ENSO series, meaning that despite shocks of any magnitude, over time ENSO returns to its long-run mean. Thus, the following linear version of the regression as in Equation (16) was estimated.

$$
\Delta E N S O_{t}=\alpha+\beta E N S O_{t-1}+\sum_{i=1}^{p-1} \theta_{i} \Delta E N S O_{t-i}+\delta^{\prime} D_{t}+\varepsilon_{t}
$$

where $E N S O_{t}$ denotes the SST anomaly used in this research, $D_{t}$ is a vector of monthly dummy variables, and the rest are parameters to be estimated.

The next step is to assess STAR-type nonlinearities in each of the equations. We use lags of the ENSO variable, $E N S O_{t-d}, d=1, \ldots, 6$, as candidate transition variables. Thus, we allow for the possibility that ENSO dynamics will vary conditional on its recent past occurrence. For example, ENSO adjustment to its long-run mean may happen at a different speed, depending on the recent conditions of the anomaly.

A battery of nonlinearity and other diagnostic test results reveal evidence of two-regime STAR-type nonlinearities throughout all considered windows (here we do not present the nonlinearity test results, for the sake of brevity, but they are available upon request). In all cases the nonlinearities appear to be of a logistic form, which constitutes the criterion for choosing the LSTAR model specification. Thus, we formulate our final 
specification as in Equation (17).

$$
\begin{aligned}
\Delta E N S O_{t} & =\alpha_{0}+\beta_{0} E_{N S O_{t-1}}+\sum_{i=1}^{4} \theta_{0, i} \Delta E N S O_{t-i}+\delta_{0}{ }^{\prime} D_{t} \\
& +\left(\alpha_{1}+\beta_{1} E N S O_{t-1}+\sum_{i=1}^{4} \theta_{1, i} \Delta E N S O_{t-i}+\delta_{1}{ }^{\prime} D_{t}\right) G(\cdot)+\varepsilon_{t}
\end{aligned}
$$

where transition function, $G(\cdot)$, is defined as Equation (18).

$$
G(\cdot)=\left\{1+\exp \left[-\frac{\gamma}{\sigma}\left(E N S O_{t-d}-c\right)\right]\right\}^{-1}
$$

The estimated parameters of the transition function suggest smooth transition between the extreme regimes. An example of the estimated transition function is presented in Figure 1. The figure illustrates a smooth transition between the extreme regimes. Note that the inflection point, $G=0.5$, of the transition function is slightly below zero, however, because of the smoothness of the transition function, we observe a continuum of inflection points, such that the normal ENSO regime can be characterized by the dynamics which is a weighted average of the dynamics underlying the two extreme regimes.

\subsection{Forecasting Results}

We use the estimated parameters and residuals from the linear and nonlinear models of each expanding window to calculate out-of-sample forecasts. We obtain ENSO forecasts using a bootstrap resampling approach. Specifically, we simulate $B$ paths of $E_{t+1 \mid t}, E_{t+2 \mid t}, \ldots, E_{t+h_{\max } \mid t}$, where $B=1000$ and $h_{\max }=36$ is the maximum horizon length. So, for a path $b$, the $h$-step-ahead forecast of ENSO is formulated as 
Equation (19).

$$
E N S O_{t+h \mid t}^{b}=f\left(E N S O_{t+h-1 \mid t}^{b}, \ldots, E N S O_{t+h-p \mid t}^{b} \mid \theta\right)+\varepsilon_{t+h \mid t}^{b}
$$

where $p$ is the autoregressive lag length of the ENSO variable, and where $E N S O_{t+h-p \mid t}^{b}=E N S O_{t+h-p \mid t}$ if $p \geq h ; \varepsilon_{t+h \mid t}^{b}$ is an innovation sampled from the pool of residuals of the estimated ENSO model. The expected forecast of ENSO, ENSO $O_{t+h \mid t}^{f b}$, is obtained by averaging the computed bootstrap paths. These forecasts are subsequently used to evaluate forecast accuracy of linear and nonlinear models using the RMSFE measures and the associated M-DM test statistics, as defined previously.

We calculate the $h$-step-ahead out-of-sample forecasts of ENSO from both the linear and nonlinear models, using estimated parameters and residuals from AR and STAR models, respectively. We then evaluate these forecasts in relation to the actual realizations of ENSO. The ultimate goal is to assess the ENSO forecast accuracy using STAR specification as compared to the linear alternative.

The first three columns of Table 1 report the RMSFE measures, along with the associated M-DM statistics, which defines the statistical significance of forecast accuracy improvement when using the nonlinear specification. We obtain these measures by applying the predicted and actual realizations of the ENSO variable to the Equations (10), and (11) - (15). These results confirm that not only does the STAR specification improve the in-sample fit, but it also outperforms the corresponding AR model in an out-of-sample setting. This improvement is apparent (statistically significant) for up to one-and-a-half year ahead forecast horizon.

We further use point forecasts of the SST anomalies to calculate percent correctly predicted (PCP) measures of ENSO events. To do so, we define El Niño and La Niña events as SST anomaly being greater than $0.5^{\circ} \mathrm{C}$ and less than $-0.5^{\circ} \mathrm{C}$, respectively. 
Columns $4-7$ of Table 1 present the PCP measures from AR and STAR models. Here as well, the relative advantage of nonlinear modelling becomes apparent at and beyond 3-months-ahead forecast horizons. Two features of interest are revealed here. First of all, the forecast horizon ENSO events is notably longer using the STAR as compared to AR models. Second, models predict La Niña events more accurately than El Niño events.

In addition to the point forecasts of the SST anomalies, we are interested in assessing forecast accuracy of the specific extreme ENSO events. We use empirical forecast densities from the bootstrap resampling procedure to compare forecast ability of the two models. In this exercise, for illustration purposes, we introduce notions of "extreme" and "moderate" events, wherein Extreme Event $\equiv S S T \geq\left|1^{\circ} \mathrm{C}\right|$;

Moderate Event $\equiv\left|1^{\circ} \mathrm{C}\right|>S S T \geq\left|0.5^{\circ} \mathrm{C}\right|$; and, finally, Normal Conditions $\equiv S S T<\left|0.5^{\circ} \mathrm{C}\right|$. Thus, for each window we forecast probabilities of each of the five events up to and including 36-months-ahead horizon. We illustrate predicted probabilities of ENSO in Figure 2. The forecasts are made using information up to and including Dec 1997 - the peak of the most extreme El Niño occurrence of the recent history. While in the very short run (1-3 months) both, linear and nonlinear models yield comparable results, the picture changes in the intermediate run (6-12 months), when the STAR model predicts La Niña episode with about 80 percent probability, while the AR model predicts La Niña with a "modest" 40 percent probability. In reality, 1997 El Niño was indeed followed by a strong La Niña in late 1998, which carried over in 1999 and 2000. This illustration is characteristic of the STAR model in that it is especially effective in predicting La Niña events in the intermediate run. 


\section{Conclusions}

ENSO is a costly phenomenon, causing considerable economic damages around the globe (Handler, 1990; Solow et al., 1998; Brunner, 2002). It has also been considered a factor in social unrests and even civil wars (Hsiang et al., 2011). Given the important nature of this climatic anomaly, researchers have attempted with some success to improve its predictability. One way to improve forecast accuracy of ENSO has been proposed to implement recent developments in nonlinear time series modelling, and specifically the smooth transition autoregressive framework of Luukkonen et al. (1988) and Terasvirta and Anderson (1992). Using the same methodology Hall et al. (2001) have found that ENSO does follow a regime-dependent dynamic process, while the regimes are connected to each other in a smooth manner. The STAR framework offers an improved in-sample fit, suggesting the possibility of improved out-of-sample fit as well.

In this research we extended the study by Hall et al. (2001) to investigate out-of-sample predicting accuracy of the ENSO phenomenon. Using root mean squared forecast error measures and modified Diebold-Mariano test statistics. We found that forecasts from the nonlinear STAR model outperform their counterparts from the linear AR model in the short and intermediate terms. These results are important for economic agents and policy makers across the world, as they offer improved ENSO forecasting and more accurate economic and social inferences. The results are also interesting from a research stand point, wherein this research is an addition to a series of studies suggesting preference of nonlinear models in ENSO forecasting. 


\section{References}

Adams, R., C. Chen, B. McCarl, and R. Weiher (1999). The Economic Consequences of ENSO Events for Agriculture. Climate Research 13(3), 165-172.

An, S. (2009). A Review of Interdecadal Changes in the Nonlinearity of the El Nino Southern Oscillation. Theoretical and Applied Climatology 97(1), 29-40.

An, S. and F. Jin (2004). Nonlinearity and Asymmetry of ENSO. Journal of Climate 17(12), 2399-2412.

Bacon, D. and D. Watts (1971). Estimating the Transition between Two Intersecting Straight Lines. Biometrika 58(3), 525 .

Berliner, L., C. Wikle, and N. Cressie (2000). Long-Lead Prediction of Pacific SSTs via Bayesian Dynamic Modeling. Journal of Climate 13(22), 3953-3968.

Boucharel, J., B. Dewitte, Y. du Penhoat, B. Garel, S. Yeh, and J. Kug (2011). ENSO Nonlinearity in a Warming Climate. Climate Dynamics 37, 2045-2065.

Brunner, A. (2002). El Nino and World Primary Commodity Prices: Warm Water or Hot Air? Review of Economics and Statistics 84(1), 176-183.

Carlson, R., D. Todey, and S. Taylor (1996). Midwestern Corn Yield and Weather in Relation to Extremes of the Southern Oscillation. Journal of Production Agriculture 9(3), 347-352.

Davies, R. (1977). Hypothesis Testing when a Nuisance Parameter is Present only under the Alternative. Biometrika 64(2), 247-254.

Davies, R. (1987). Hypothesis Testing when a Nuisance Parameter is Present only under the Alternative. Biometrika 74(1), 33-43. 
Deng, Z. and Y. Tang (2009). The Retrospective Prediction of ENSO from 1881 to 2000 by a Hybrid Coupled Model: (II) Interdecadal and Decadal Variations in Predictability. Climate Dynamics 32(2), 415-428.

Diebold, F. and R. Mariano (1995). Comparing Predictive Accuracy. Journal of Business 83 Economic Statistics 13(3), 253-263.

Eitrheim, O. and T. Terasvirta (1996). Testing the Adequacy of Smooth Transition Autoregressive Models. Journal of Econometrics 74(1), 59-75.

Glantz, M. (2001). Currents of Change: Impacts of El Niño and La Niña on Climate and Society. Cambridge Univ Pr.

Granger, C. and T. Terasvirta (1993). Modelling Nonlinear Economic Relationships. Oxford University Press, USA.

Halide, H. and P. Ridd (2008). Complicated ENSO Models Do Not Significantly Outperform Very Simple ENSO Models. International Journal of Climatology 28(2), 219-233.

Hall, A., J. Skalin, and T. Teräsvirta (2001). A Nonlinear Time Series Model of El Nińo. Environmental Modelling and Software 16(2), 139-146.

Hamilton, J. D. (1989). A New Approach to the Economic Analysis of Nonstationary Time Series and the Business Cycle. Econometrica 57(2), 357-384.

Handler, P. (1990). USA Corn Yields, The El Niño and Agricultural Drought: 1867-1988. International Journal of Climatology 10(8), 819-828.

Hansen, J., A. Hodges, and J. Jones (1998). ENSO Influences on Agriculture in the Southeastern United States. Journal of Climate 11(3), 404-411. 
Harvey, D., S. Leybourne, and P. Newbold (1997). Testing the Equality of Prediction Mean Squared Errors. International Journal of Forecasting 13(2), 281-291.

Hsiang, S., K. Meng, and M. Cane (2011). Civil Conflicts are Associated with the Global Climate. Nature 476, 438-441.

Keppenne, C. (1995). An ENSO Signal in Soybean Futures Prices. Journal of Climate 8(6), 1685-1689.

Kim, M. and B. McCarl (2005). The Agricultural Value of Information on the North Atlantic Oscillation: Yield and Economic Effects. Climatic Change 71(1), 117-139.

Kirtman, B. and P. Schopf (1998). Decadal Variability in ENSO Predictability and Prediction. Journal of Climate 11(11), 2804-2822.

Kuan, C.-M. and H. White (1994). Artificial Neural Networks: An Econometric Perspective. Econometric Reviews 13(1), 1-91.

Legler, D., K. Bryant, and J. O'Brien (1999). Impact of ENSO-related Climate Anomalies on Crop Yields in the U.S. Climatic Change 42(2), 351-375.

Lundbergh, S. and T. Terasvirta (2004). Forecasting with Smooth Transition Autoregressive Models. In M. P. Clements and D. F. Hendry (Eds.), A Companion to Economic Forecasting, pp. 485-509. Blackwell Publishing.

Luukkonen, R., P. Saikkonen, and T. Terasvirta (1988). Testing Linearity Against Smooth Transition Autoregressive Models. Biometrika 75(3), 491-499.

Milas, C. and P. Rothman (2008). Out-of-Sample Forecasting of Unemployment Rates with Pooled STVECM Forecasts. International Journal of Forecasting 24(1), 101-121. 
Newey, W. and D. Kenneth (1987). A Simple, Positive Semi-definite, Heteroskedasticity and Autocorrelation Consistent Covariance Matrix. Econometrica 55(3), 703-708.

Pielke Jr, R. and C. Landsea (1999). La Nina, El Nino, and Atlantic Hurricane Damages in the United States. Bulletin of the American Meteorological Society 80(10), $2027-2034$.

Sarantis, N. (1999). Modeling Non-linearities in Real Effective Exchange Rates. Journal of International Money and Finance 18(1), 27-45.

Skalin, J. and T. Terasvirta (2002). Modeling Asymmetries and Moving Equilibria in Unemployment Rates. Macroeconomic Dynamics 6(2), 202-241.

Solow, A., R. Adams, K. Bryant, D. Legler, J. O’Brien, B. McCarl, W. Nayda, and R. Weiher (1998). The Value of Improved ENSO Prediction to US Agriculture. Climatic Change 39(1), 47-60.

Swanson, N. (1998). Money and Output Viewed through a Rolling Window. Journal of Monetary Economics 41(3), 455-473.

Tang, Y. and W. Hsieh (2002). Hybrid Coupled Models of the Tropical Pacific: II ENSO prediction. Climate dynamics 19(3), 343-353.

Tangang, F., B. Tang, A. Monahan, and W. Hsieh (1998). Forecasting ENSO Events: A Neural Network - Extended EOF Approach. Journal of Climate 11(1), 29-41.

Terasvirta, T. (1994). Specification, Estimation, and Evaluation of Smooth Transition Autoregressive Models. Journal of the American Statistical Association 89(425), $208-218$. 
Terasvirta, T. (1995). Modelling Nonlinearity in US Gross National Product 1889-1987. Empirical Economics 20(4), 577-597.

Terasvirta, T. and H. Anderson (1992). Characterizing Nonlinearities in Business Cycles using Smooth Transition Autoregressive Models. Journal of Applied Econometrics 7, S119-S136.

Terasvirta, T., D. Van Dijk, and M. Medeiros (2005). Linear Models, Smooth Transition Autoregressions, and Neural Networks for Forecasting Macroeconomic Time Series: A Re-examination. International Journal of Forecasting 21(4), 755-774.

Tong, H. (1990). Non-linear Time Series: A Dynamical System Approach. Oxford University Press.

Tsay, R. (1989). Testing and Modeling Threshold Autoregressive Processes. Journal of the American Statistical Association 84(405), 231-240.

Ubilava, D. (2012). El Niño, La Niña, and World Coffee Price Dynamics. Agricultural Economics 43(1), 17-26. 


\section{$5 \quad$ Figures}
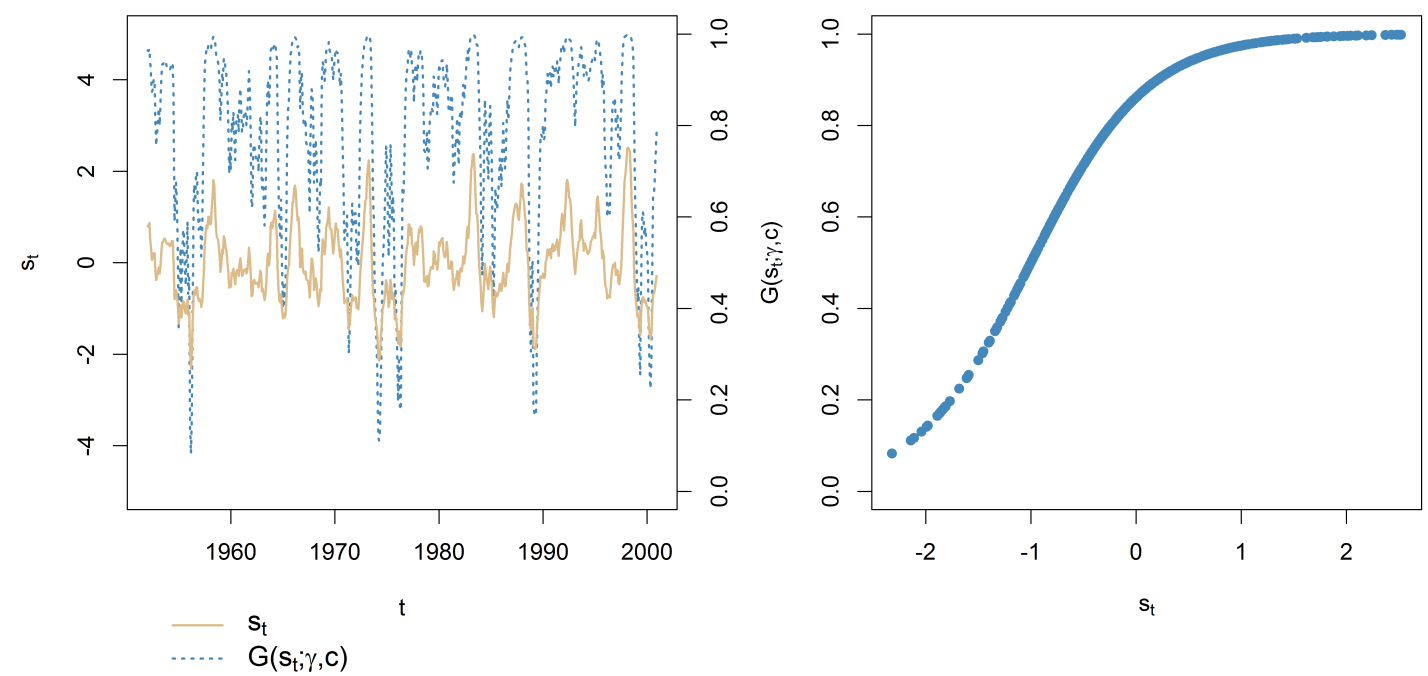

Figure 1: Estimated Transition Function (January 1952 - December 2000) 



Figure 2: Predicted Probabilities of ENSO Events (December 1997) 


\section{Tables}

Table 1: ENSO Forecasting and Evaluation

\begin{tabular}{|c|c|c|c|c|c|c|c|}
\hline \multirow[t]{2}{*}{$\overline{\mathrm{h}}$} & \multirow[t]{2}{*}{ RMSFE $_{\mathrm{AR}}$} & \multirow[t]{2}{*}{ RMSFE $_{\mathrm{STAR}}$} & \multirow[t]{2}{*}{$\mathrm{RE}_{\mathrm{STAR} / \mathrm{AR}}$} & \multicolumn{2}{|c|}{ AR } & \multicolumn{2}{|c|}{ STAR } \\
\hline & & & & La Niña & El Niño & La Niña & El Niño \\
\hline 1 & 0.207 & 0.201 & $0.967 *$ & 91.3 & 87.0 & 93.5 & 87.0 \\
\hline 2 & 0.376 & 0.348 & $0.924 * * *$ & 70.2 & 72.1 & 68.1 & 72.1 \\
\hline 3 & 0.506 & 0.453 & $0.897^{* * *}$ & 58.3 & 57.4 & 58.3 & 67.6 \\
\hline 4 & 0.607 & 0.534 & $0.880 * * *$ & 42.9 & 47.1 & 53.1 & 63.2 \\
\hline 5 & 0.694 & 0.604 & $0.871 * * *$ & 36.7 & 36.8 & 44.9 & 54.4 \\
\hline 6 & 0.758 & 0.656 & $0.865 * * *$ & 24.5 & 22.4 & 36.7 & 44.8 \\
\hline 7 & 0.808 & 0.700 & $0.866^{* * *}$ & 18.4 & 13.4 & 36.7 & 38.8 \\
\hline 8 & 0.843 & 0.735 & $0.872 * * *$ & 12.2 & 10.4 & 38.8 & 28.4 \\
\hline 9 & 0.864 & 0.765 & $0.885^{* * *}$ & 6.1 & 4.5 & 32.7 & 17.9 \\
\hline 10 & 0.874 & 0.786 & $0.899 * * *$ & 4.1 & - & 28.6 & 9.0 \\
\hline 11 & 0.879 & 0.800 & $0.910 * *$ & 2.0 & - & 28.6 & 1.5 \\
\hline 12 & 0.882 & 0.809 & $0.918 * *$ & 2.0 & - & 24.5 & - \\
\hline 13 & 0.882 & 0.811 & $0.918^{* * *}$ & - & - & 18.4 & - \\
\hline 14 & 0.882 & 0.810 & $0.919 * * *$ & - & - & 16.3 & - \\
\hline 15 & 0.879 & 0.812 & $0.924 * * *$ & - & - & 14.3 & - \\
\hline 16 & 0.875 & 0.821 & $0.938 * * *$ & - & - & 12.2 & - \\
\hline 17 & 0.870 & 0.831 & $0.956 * *$ & - & - & 10.2 & - \\
\hline 18 & 0.863 & 0.841 & 0.974 & - & - & 8.2 & - \\
\hline 19 & 0.859 & 0.850 & 0.989 & - & - & 6.1 & - \\
\hline 20 & 0.858 & 0.859 & 1.002 & - & - & 2.0 & - \\
\hline 21 & 0.859 & 0.865 & 1.007 & - & - & - & - \\
\hline 22 & 0.863 & 0.869 & 1.007 & - & - & - & - \\
\hline 23 & 0.867 & 0.871 & 1.005 & - & - & - & - \\
\hline 24 & 0.870 & 0.872 & 1.002 & - & - & - & - \\
\hline 25 & 0.873 & 0.872 & 0.999 & - & - & - & - \\
\hline 26 & 0.879 & 0.875 & 0.995 & - & - & - & - \\
\hline 27 & 0.884 & 0.876 & 0.992 & - & - & - & - \\
\hline 28 & 0.887 & 0.878 & $0.990 *$ & - & - & - & - \\
\hline 29 & 0.886 & 0.878 & $0.991 *$ & - & - & - & - \\
\hline 30 & 0.884 & 0.876 & $0.991 *$ & - & - & - & - \\
\hline 31 & 0.883 & 0.877 & 0.993 & - & - & - & - \\
\hline 32 & 0.882 & 0.877 & 0.994 & - & - & - & - \\
\hline 33 & 0.882 & 0.876 & 0.994 & - & - & - & - \\
\hline 34 & 0.881 & 0.875 & 0.993 & - & - & - & - \\
\hline 35 & 0.880 & 0.875 & 0.993 & - & - & - & - \\
\hline 36 & 0.881 & 0.876 & 0.994 & - & - & - & - \\
\hline
\end{tabular}

Note: ${ }^{*},{ }^{* *}$ and ${ }^{* * *}$ denote statistical significance of M-DM tests at $0.10,0.05$ and 0.01 levels, respectively; entries in the column headed with $h$ denote forecast horizon; entries in the columns headed with $\mathrm{RMSFE}_{A R}$ and RMSFE $_{S T A R}$ denote RMSFE measures of AR and STAR models, while RMSFE $\mathrm{R}_{S T A R / A R}$ denotes a ratio of $\mathrm{RMSFE}_{S T A R}$ and $\mathrm{RMSFE}_{A R}$; entries in the columns headed with La Niña and El Niño denote percent correctly predicted measures of each respective ENSO event. 